\title{
Maternal Stress During Pregnancy and Allergic Diseases in Children During the First Year of Life
}

\author{
Katarzyna Smejda MD PhD, Kinga Polanska PhD, Dorota Merecz-Kot PhD, Anna Krol MPH, \\ Wojciech Hanke MD PhD, Joanna Jerzynska MD PhD, Wlodzimierz Stelmach MD PhD, \\ Paweł Majak MD PhD, and Iwona Stelmach MD PhD
}

\begin{abstract}
BACKGROUND: Many recent studies indicate that prenatal maternal distress increases the risk of allergic diseases in children. The mechanisms that favor it are still unclear. OBJECTIVES: We aimed to assess the association between exposure to different kinds of prenatal stress and the occurrence of atopic dermatitis, food allergy, wheezing, and recurrent respiratory tract infections in children. METHODS: The study population consisted of $\mathbf{3 7 0}$ mother-child pairs from a Polish Mother and Child Cohort (REPRO_PL). The analysis was restricted to the women who worked at least one month during the pregnancy period. Maternal psychological stress during pregnancy was assessed based on the Subjective Work Characteristics Questionnaire, the Perceived Stress Scale, and the Social Readjustment Rating Scale. The presence of atopic dermatitis, food allergy, wheezing, and recurrent respiratory tract infections in children was evaluated by doctors at 12 months of age. RESULTS: In a univariate model, we showed significant association between maternal life stress (according to the Perceived Stress Scale) and stressful life events (according to the Social Readjustment Rating Scale) and infant wheezing (at least 1 episode of wheezing during the first year of life). A multivariate model of logistic regression analysis revealed that maternal stress during pregnancy, described by the Social Readjustment Rating Scale, increased the risk of wheezing in children (OR 1.09, 95\% CI 1.01-1.02) independently from other predictors of wheezing previously determined in this cohort, such as the number of infections and maternal smoking. We observed also significant positive association between maternal life stress during pregnancy measured by the Perceived Stress Scale and the risk of recurrent respiratory tract infections in the first year of life, however it was not significant after adjustment for confounding variables. CONCLUSIONS: Maternal stress during pregnancy increases the risk of childhood wheezing. The effects of stress during pregnancy on the onset of allergic diseases in children should be developed and translated into early prevention strategies. Key words: maternal stress; children; atopic dermatitis; wheezing; food allergy; recurrent respiratory tract infections. [Respir Care 2018;63(1):70-76. (C) 2018 Daedalus Enterprises]
\end{abstract}

\section{Introduction}

The natural history of atopic diseases begins in utero. The onset of atopic diseases may be the result of heritable

Drs Smejda, Jerzynska, and I. Stelmach are affiliated with the Department of Pediatrics and Allergy, Medical University of Lodz, Copernicus Memorial Hospital in Lodz, Poland. Drs Polanska and Hanke, as well as Ms. Krol, are affiliated with the Department of Environmental Epidemiology, Nofer Institute of Occupational Medicine, Lodz, Poland. Dr MereczKot is affiliated with the Department of Health and Work Psychology, Nofer Institute of Occupational Medicine, Lodz, Poland; Dr W. Stelmach is affiliated with the Department of Social and Preventive Medicine, Medical University of Lodz, Lodz, Poland. Dr Majak is affiliated with changes of gene expression, without any alteration in DNA sequences occurring in response to early environmental

\footnotetext{
the Department of Internal Medicine, Asthma and Allergy, Medical University of Lodz, Lodz, Poland.
}

The authors have disclosed no conflicts of interest.

Correspondence: Iwona Stelmach MD PhD, Department of Pediatrics and Allergy, Medical University of Lodz, Poland, Copernicus Memorial Hospital, Korczak Paediatric Center, Piłsudskiego 71 Str, 90-329 Lodz, Poland. E-mail: alergol@kopernik.lodz.pl.

DOI: $10.4187 /$ respcare. 05692 
stimuli. ${ }^{1}$ Prenatal stress has been associated with asthma or wheezing in several studies. ${ }^{2-8}$ Chiu et $\mathrm{al}^{2}$ showed that prenatal or postnatal negative life events alone both showed an exposure-response relationship between higher stress and child wheezing; ie, associations between high prenatal stress and wheezing were significant in children born to nonsensitized mothers but not in the sensitized group. Wright et $\mathrm{al}^{3}$ showed that maternal prenatal cortisol disruption and obesity were independently associated with childhood wheezing. Guxens et $\mathrm{al}^{5}$ found that mothers with psychological distress during pregnancy had increased odds that their children would have episodes of wheezing from 1 to $4 \mathrm{y}$ of age. According to Peters et al, ${ }^{8}$ higher prenatal maternal stress was associated with increased cord blood immunoglobulin $\mathrm{E}(\mathrm{IgE})$. Medsker et $\mathrm{al}^{9}$ found that perinatal stress may cause changes in glucocorticoid and cytokine production, leading to preterm labor; in turn, changes in immune responses and lung development due to both stress and prematurity place these children at increased risk of asthma. Moreover, maternal stress can alter fetal cytokine balance, favoring $\mathrm{T}$ helper (TH2) (allergic) immune responses characteristic of atopic asthma: interleukin 6 (IL-6), which has been associated with premature labor, can promote $\mathrm{TH} 2$ responses by stimulating production of IL-4 and IL-13. ${ }^{9}$ Maternal depression could affect child outcomes through altered placental function, epigenetic changes in the child, and stress reactivity. ${ }^{10}$ Prenatal stress during the third trimester of pregnancy is associated with increased methylation of the glucocorticoid receptor gene $(N R 3 C 1)^{11}$ and increased cortisol responses in infants at 3 months postpartum. Cortisol can downregulate the hypothalamic-pituitary-adrenal axis by binding to glucocorticoid receptors and mineralocorticoid receptors. During pregnancy, the fetus is exposed to endogenous glucocorticoids from the maternal circulation and, potentially, to exogenous glucocorticoids used to treat (eg, pre-term labor, maternal asthma, or autoimmune disorders). This is important, because prenatal exposure to increased levels of glucocorticoids (endogenous or exogenous) can cause lasting alterations in the fetal and neonatal hypothalamic-pituitary-adrenal axis. ${ }^{12}$

Considering the early onset of atopic dermatitis, which most often arises in the first year of life, risk factors occurring very early in life must be considered. ${ }^{13}$ The pathogenesis of atopic dermatitis is complex and is still poorly understood. In addition to genetic predisposition attributed to immune dysregulation and hypersensitivity, development and continuation of atopic dermatitis are thought to be associated with environmental and psychological triggers and skin barrier defects. ${ }^{1,14,15}$

Maternal distress increases the predicted probability of atopic dermatitis. A recent systematic review showed a relationship between prenatal maternal stress, such as negative life events, anxiety/depression, bereavement, distress,

\section{QUICK LOOK}

\section{Current knowledge}

The natural history of atopic diseases begins in utero. The effect of maternal stress during pregnancy on atopic disorders in the offspring is a matter of debate. Although there have been some studies about the association between exposure to stress during pregnancy and the prevalence of atopic dermatitis, food allergy, and respiratory symptoms during the first year of life, the results are not clear.

\section{What this paper contributes to our knowledge}

Premature stress increased the risk of wheezing, but it did not influence the risk of atopic dermatitis and food allergy in the first year of life. There also appeared to be no correlation between occupational maternal stress during pregnancy and allergic diseases in children.

and job strain, and atopic disorders in the child in most studies. ${ }^{16}$ Psychological stress causes abnormal skin-barrier function in humans, ${ }^{17}$ and it induces an increase in endogenous corticosteroids, which in turn appears to disrupt not only barrier function but also stratum corneum cohesion as well as epidermal antimicrobial defense. ${ }^{18}$ During pregnancy, increased oxygen demand augments the rate of production of reactive oxygen species, and women, even during normal pregnancies, experience elevated serum oxidative stress (OS) levels. ${ }^{1,19,20}$ OS can directly cause damage to epidermal keratinocytes by DNA damage, to cellular enzymes, and to cell membrane structures through lipid oxidation. ${ }^{1}$ It is also suggested that gestational exposure to maternal stress alters the development of humoral immune competence in the fetus and the hormonal and immunological responses to stress in postnatal life, thus implicating a risk of developing atopic disorders. ${ }^{20,21}$ The aim of the present study was to assess the association between exposure to different kinds of prenatal stress and the occurrence of atopic dermatitis, food allergy, wheezing, and recurrent respiratory tract infections in children.

\section{Methods}

\section{Study Design and Population}

The mother-child pairs included in this study were part of the Polish Mother and Child Cohort (REPRO_PL), a multi-center prospective cohort established in 2007 to evaluate environmental factors contributing to pregnancy outcomes and children's health and neurodevelopment. ${ }^{23-25}$ All data were prospectively collected. Women were re- 
cruited if they fulfilled the following inclusion criteria: single pregnancy up to 12 weeks of gestation, no assisted conception, no pregnancy complications, and no chronic diseases as specified in the study protocol. ${ }^{23,25}$ The whole cohort comprises 1,700 mother-child pairs followed from pregnancy to $2 \mathrm{y}$ of age. ${ }^{26}$

The participants were interviewed once in each trimester of pregnancy to collect and update sociodemographic data, medical and reproductive history, and information about environmental, occupational, and lifestyle factors. Children were assessed for their exposure and health status at $1 \mathrm{y}$ of age. $24,27-30$

Taking into account the financial and organizational feasibility of the study, this assessment is restricted to a subpopulation from the Lodz district (central Poland). We intended to assess the impact of prenatal stress in the development of atopic dermatitis, food allergy, wheezing, and recurrent infections in children, considering occupational stress, perceived levels of stress in daily life, and the occurrence of stressful life events. Therefore, the analysis was restricted to the population of mothers working at least one month during pregnancy (464 out of 547; 85\% of the population). Complete data on stress during pregnancy were available for 370 of the 464 women who indicated being employed during pregnancy.

The study was approved by the Ethical Committee of the Nofer Institute of Occupational Medicine, Lodz, Poland; written consent was obtained from all the subjects before the study. The study was supported by the DEC2014/15/B/NZ7/00998 grant from the National Science Centre.

\section{Assessment of Maternal Stress During Pregnancy}

Between week 20 and week 24 of gestation, the subjects completed 2 self-administered questionnaires to measure occupational stress and life stress. A third questionnaire measuring life-events stress was completed between weeks 30 and 34 of gestation. The questionnaires were chosen to cover a wide scope of potential stress sources.

Occupational stress was assessed using the Subjective Work Characteristics Questionnaire. ${ }^{31}$ This widely used scale for diagnosis of occupational stress in Poland consists of 55 items describing potential occupational stressors (the following conditions were evaluated: general work stress, work overload, lack of rewards, uncertainty in workplace, social relations in workplace, threat, physical burdens, unpleasant work conditions, lack of control, lack of support, responsibility). The subjects were asked to state whether a given characteristic is present in their work position and how stressful they find it to be. Each item had the following answers: 1) a given psychosocial factor is not present; 2 ) it is present but it is neither irritating nor disturbing; 3 ) it is irritating or disturbing from time to time; 4) it is quite often irritating or disturbing; and 5) it is irritating all the time. The general indicator of the level of stress was the sum of points marked by each subject (the higher the score, the stronger the perceived job stress). In our analysis, an additional indicator of occupational stress, the number of stressful work characteristics as pointed out by each subject, was used.

Life stress was assessed by means of the Perceived Stress Scale (PSS), a 10-item scale that measures an individual's level of perceived stress in the past month. ${ }^{32}$ The items are designed in such a way to tap into how unpredictable, uncontrollable, and overloaded the subjects find life to be. The scale also includes a number of direct queries about current levels of experienced stress. The response options for each item indicate the frequency with which it occurs, from 0 , meaning never, to 4 , meaning very often. The results were the sum of points. The higher the score obtained by the subjects, the higher the level of their global stress.

Life events as stress factors were measured by a modified version of the Social Readjustment Rating Scale (SRRS). ${ }^{33}$ This scale is based on the observation that important life changes, whether positive, such as marriage or negative, such as death of a close friend, induce stress. Not only was the presence of critical life events during pregnancy measured, but its impact on the subjects' emotional well-being was also assessed. Thus, we obtained 2 indices of stress: a sum of impact scores assigned to the pointed life events and an individual perception of stress related to indicated events, which was the sum of the scores calculated from the answer to the question, "To what extent did the event have impact on your emotional well-being?" Answers ranged from "none" to "extreme impact" (ie, 0-3).

Within the two scales selected above, different measures of stress, both objective and subjective, were used: the burden of life events that appeared in the lives of pregnant women (objectified, intersubjective measure of stress according to SRRS); and the perceived, subjectively assessed level of stress (according to PSS and the additional module of SRRS, where subjective stressfulness of the listed life events was assessed by pregnant women).

\section{Child Health Assessment}

Children's health status was assessed at around $1 \mathrm{y}$ of age (range, $10-18 \mathrm{mo}$ ). For the appropriate recognition of a child's health status, an interview was performed with each mother and supplemented or verified with information from the medical chart of each child. ${ }^{24,27-30}$ In addition, the current child health status assessment was performed by a pediatrician/allergist. At that time, information concerning sociodemographic, environmental, and lifestyle factors were also updated by conducting interviews with mothers. Based on availability of data on prenatal stress 
exposure and physical examination at the age of 12 months, the analysis was performed for 370 children.

The first part of the questionnaire covered certain sociodemographic information (ie, family size, material status of the family, and parental educational level). The occurrence of allergy among family members was noted. The second part of the questionnaire (developed by an allergist and based on recommendations from the International Study of Asthma and Allergies in Childhood) investigated the child's health and condition. The respiratory outcomes were analyzed for symptoms of wheezing, cough, bronchitis, eczema, and food allergy. The duration of each infection and disease, medications taken, and hospitalizations, if any, were identified. Recurrent respiratory infection was defined as doctor-diagnosed viral/bacterial lower airway infections occurring $\geq 2$ times per year.

\section{Statistical Analysis}

The associations of prenatal stress with atopic dermatitis, food allergy, wheezing, and recurrent respiratory tract infections were assessed with logistic regression. First, logistic regression was used to assess the relationship between dependent variables and each single-stress scale. In the second step, significant associations were corrected for the effect of independent risk factors for each of the outcomes (previously described in the same cohort). ${ }^{27-30} \mathrm{~A}$ stepwise forward procedure was then used to select variables. Predictors with $P$ values of at least .1 estimated in univariate models were included in multivariate regression analyses. All of the statistical analyses were performed using STATISTICA 12.0 (StatSoft, Inc., Tulsa, Oklahoma). The null hypothesis was rejected if $P<.05$.

\section{Results}

Our analysis included 370 children and their parents. Baseline characteristics of the population are given in Table 1 . We noted that wheezing in our cohort appeared in $<10 \%$ of children, which is similar with the prevalence reported for infants in our country.

Descriptive statistics of stress measures are given in Table 2. All scales were characterized by skewed distribution, so they were log-transformed before inclusion in logistic regression analyses. Risk factors of all outcomes were previously characterized in the same cohort. Independent risk factors were included as a confounders in the current analysis. Logistic regression analysis did not show any significant associations between exposure and the prevalence of atopic dermatitis or food allergy (Table 3). In the univariate analysis we found a significant association between two different stress scales (PSS and SRRS) and wheezing (at least 1 episode of wheezing during the first year of life) (Table 3).
Table 1. Description of the Study Cohort

\begin{tabular}{|c|c|}
\hline \multicolumn{2}{|l|}{ Clinical profile } \\
\hline Atopic dermatitis & $43(11.6)$ \\
\hline Food allergy & $56(15.1)$ \\
\hline Wheezing & $36(9.7)$ \\
\hline Respiratory tract infection ( $\geq 2$ episodes) & $119(32.2)$ \\
\hline \multicolumn{2}{|l|}{ Family status } \\
\hline Mother's age, $y$, mean $\pm \mathrm{SD}$ & $29.1 \pm 4.3$ \\
\hline$\leq 24$ & $55(14.9)$ \\
\hline $25-29$ & $115(31.1)$ \\
\hline $30-34$ & $157(42.2)$ \\
\hline$\geq 35$ & $43(11.6)$ \\
\hline \multicolumn{2}{|l|}{ Maternal education } \\
\hline$<9$ y of education & $6(1.6)$ \\
\hline $10-12$ y of education & $99(26.8)$ \\
\hline$>12 \mathrm{y}$ of education & $265(71.6)$ \\
\hline Father's age, $y$, mean $\pm S D$ & $31.1 \pm 5.6$ \\
\hline \multicolumn{2}{|l|}{ Paternal education } \\
\hline$<9$ y of education & $19(5.1)$ \\
\hline $10-12$ y of education & $193(52.2)$ \\
\hline$>12 \mathrm{y}$ of education & $158(42.7)$ \\
\hline Marital status (unmarried) & $84(22.7)$ \\
\hline Maternal smoking at the first trimester of pregnancy & $40(10.8)$ \\
\hline Parental atopy & $143(38.6)$ \\
\hline \multicolumn{2}{|l|}{ Socioeconomic status } \\
\hline Low & $31(8.4)$ \\
\hline Medium & $250(67.6)$ \\
\hline High & $83(22.4)$ \\
\hline Alcohol during pregnancy & $30(8.1)$ \\
\hline
\end{tabular}

Multivariate model of logistic regression analysis revealed that premature stress, according to SRRS, increased the risk of wheezing (OR 1.09, 95\% CI 1.01-1.02) independently from other predictors of wheezing previously determined in this cohort, such as the number of infections and maternal smoking. We also observed a significant positive association between perceived stress in mothers during pregnancy and the risk of recurrent respiratory tract infections in the first year of life (Table 3). This association, however, was not significant after adjustment with statistical predicators of this outcome determined in this cohort previously.

\section{Discussion}

In the literature, there are studies suggesting that prenatal maternal distress increases the risk of allergic diseases in offspring. ${ }^{2-8,16}$ In our study, we found that premature stress, described by life events as stress factors (according to SRRS), increased the risk of wheezing independently from other predictors of wheezing, such as the number of infections and paternal smoking. Our study 
Table 2. Descriptive Statistics of Exposure Variables

\begin{tabular}{lcrrr}
\hline \multicolumn{1}{c}{ Stress Scores } & $n$ & Median & Lower Quartile & Higher Quartile \\
\hline PSS & 370 & 17 & 14 & 21 \\
SRRS total score & 345 & 2 & 0 & 130 \\
SRRS sum of wages & 345 & 76 & 73 & 107 \\
SWCQ sum in points & 330 & 89 & 14 & 28 \\
SWCQ number of psychosocial factors at work & 330 & & & \\
& & & \\
PSS = Perceived Stress Scale & & & \\
SRRS = Social Readjustment Rating Scale & & & \\
SWCQ = Subjective Work Characteristics Questionnaire & &
\end{tabular}

Table 3. Associations Between Atopic Dermatitis, Food Allergy, Wheezing, Recurrent Respiratory Tract Infections, and Stress Measures During Pregnancy

\begin{tabular}{|c|c|c|c|c|c|c|c|c|}
\hline & \multicolumn{2}{|c|}{ Atopic Dermatitis } & \multicolumn{2}{|c|}{ Food Allergy } & \multicolumn{2}{|l|}{ Wheezing } & \multicolumn{2}{|c|}{$\begin{array}{l}\text { Recurrent Respiratory } \\
\text { Tract Infection }\end{array}$} \\
\hline & OR $(95 \% \mathrm{CI})$ & $P$ & OR $(95 \% \mathrm{CI})$ & $P$ & OR $(95 \% \mathrm{CI})$ & $P$ & OR $(95 \% \mathrm{CI})$ & $P$ \\
\hline PSS & $0.98(0.93-1.04)$ & .45 & $0.97(0.93-1.02)$ & .30 & $1.081(1.017-1.149)$ & .01 & $1.05(1.01-1.09)$ & .03 \\
\hline SRRS total score & $0.99(0.92-1.06)$ & .74 & $1.02(0.96-1.08)$ & .63 & $1.099(1.026-1.177)$ & .01 & $1.02(0.97-1.07)$ & .49 \\
\hline SRRS sum of wages & $1.00(1.00-1.00)$ & .87 & $1.00(1.00-1.00)$ & .78 & $1.005(1.001-1.010)$ & .03 & $1.00(1.00-1.00)$ & .56 \\
\hline SWCQ sum in points & $0.99(0.98-1.01)$ & .040 & $1.00(0.98-1.01)$ & .46 & $0.998(0.982-1.015)$ & .84 & $1.01(1.00-1.02)$ & .33 \\
\hline $\begin{array}{l}\text { SWCQ no. } \\
\text { psychosocial factors }\end{array}$ & $0.98(0.94-1.02)$ & .22 & $0.98(0.94-1.01)$ & .17 & $1.010(0.971-1.051)$ & .62 & $1.01(0.99-1.04)$ & .40 \\
\hline
\end{tabular}

psychosocial factors at work

The data were calculated using a univariate model of logistic regression analysis

PSS $=$ Perceived Stress Scale;

SRRS $=$ Social Readjustment Rating Scale

SWCQ $=$ Subjective Work Characteristics Questionnaire

also showed positive, but not statistically significant, association between perceived stress (according to PSS) in mothers during pregnancy and the risk of recurrent respiratory tract infections in the first year of life. We didn't find any correlation between occupational maternal stress during pregnancy and atopic diseases in children or between maternal stress during pregnancy and other allergic diseases such as atopic dermatitis and food allergy in children.

Psychological stress during pregnancy increases the risk of childhood wheezing and asthma. The influence of exposure timing has not been completely elucidated. Trump et $\mathrm{al}^{34}$ concluded that high maternal stress was associated with an increased risk for persistent wheezing until the age of $5 \mathrm{y}$, while de Marco et $\mathrm{al}^{7}$ observed in large populations that $9 \%$ of the mothers experienced stressful life events during pregnancy (excluding divorce, mourning, or loss of employment). Their children had a statistically significantly higher lifetime prevalence of wheezing (31.6\% vs $23.1 \%)$, asthma (8.9\% vs $5.6 \%)$, allergic rhinitis ( $10.9 \%$ vs $7.3 \%)$, and atopic eczema $(29.7 \%$ vs $21.1 \%)$ than those of mothers without stressful life events during pregnancy (excluding divorce, mourning, or loss of employment). ${ }^{7}$ Others had similar results. Hartwig et $\mathrm{al}^{35}$ showed that maternal adverse life events during the second half of gestation are linked to an increased risk for the development of atopic disorders, asthma, and eczema. Liu et $\mathrm{al}^{36}$ showed that prenatal stress is possibly associated with asthma events in children aged $0-3$ y, but not with asthma in children $4-15$ y old, irrespective of age at asthma onset. Rosa et $\mathrm{al}^{37}$ showed that prenatal and postnatal stress (assessed by life events) in mothers was associated with wheezing in preschoolaged children, and the effect of postnatal stress was stronger in girls. Our study confirms these results related to wheezing.

Numerous studies have shown that maternal stress during pregnancy may be associated with the development of respiratory allergy, but there are only a few studies on food allergy. ${ }^{28,35,38}$ Polloni et $\mathrm{al}^{38}$ found that maternal stress during the perinatal period may increase the risk of food allergy in a child. We found no correlation in our study between maternal stress and food allergy. Therefore, further studies are needed to explain the role of early stress in the pathogenesis of food allergy.

There are few studies on the impact of stress related to work on allergic diseases in children. This could be in part because most pregnant women are on medical leave and do not work. Larsen et al ${ }^{39}$ showed that maternal exposure to self-reported high job strain during pregnancy was associated with $15 \%$ higher odds of atopic dermatitis among 
7-y-old children. Furthermore, an association between mothers with active jobs and asthma among 7-y-old children was found. ${ }^{39}$ Sidbury et al ${ }^{14}$ showed that, in addition to work stress, working in professional or technical occupations during pregnancy increased the risk of childhood atopic dermatitis. The mothers of children with atopic dermatitis had a longer working time than those without atopic dermatitis. ${ }^{14} \mathrm{We}$ found no correlation in our study between occupational maternal stress during pregnancy and atopic diseases in children.

The mechanisms by which stress may affect the development of atopic diseases are not fully understood. Chang et $\mathrm{al}^{19}$ showed that prenatal distress decreased placental glutathione to glutathione disulfide ratios and, especially in those who later had atopic dermatitis, decreased placental $11 \beta$-hydroxysteroid dehydrogenase type 2 levels and increased IgE levels at $1 \mathrm{y}$ of age. Wen et $\mathrm{al}^{40}$ concluded that elevated cord blood IgE, LT- $\alpha$ and FceRI- $\beta$ genotypes, and maternal stress during pregnancy were associated with having physician-diagnosed atopic dermatitis in 2-y-old children and increased the predictive ability for atopic dermatitis after taking into account gender, maternal education, and parental atopic history. Karlsson et $\mathrm{al}^{41}$ observed positive correlations between concentrations of potentially proallergenic cytokines and maternal prenatal psychological symptoms. The concentrations of TH2-related IL-9 and IL-13 and TH1-related IL-12 correlated positively with prenatal depressive symptoms. Higher interferon (IFN)- $\gamma /$ IL-4 ratio and TH2-related IL-5 concentration correlated positively with depressive symptoms. Pregnancy-related anxiety score correlated positively with IL-12, IL-13, and anti-inflammatory IL-10 concentrations. Tsuji et $\mathrm{al}^{42}$ supports the hypothesis that maternal psychological stress affects the inflammatory response in their allergic children. They found significant relationships between maternal psychological stress and IL-6 and IL-8 mRNA expressions in children with asthma and allergic rhinitis. Deregulated neuroendocrine and neurotransmitter receptor interactions were observed in stressed mothers and their children. ${ }^{34}$

Our study has several limitations. Although we have measured different aspects of stress, we did not cover maternal anxiety, depression, or stress related to pregnancy and performing parental roles. We cannot exclude the possibility that confounding by unmeasured risk factors produced associations between exposure of interest and a child's health status. In addition, the questionnaires measuring stress were applied during the second and third trimesters of pregnancy, so the exact time of exposure allowing for assessment of critical windows was not possible. Finally, we didn't measure maternal stress before 12 weeks of gestation, so we can't conclude whether that period was essential for development of our infants.
In summary, we found a significant association between prenatal exposure to maternal stress measured by life events as stress factors and wheezing in children. To further understand and quantify the effects of prenatal stress on child allergic diseases, additional studies are needed. Understanding the effects of stress during pregnancy may better inform prevention strategies.

\section{REFERENCES}

1. Ji H, Li XK. Oxidative stress in atopic dermatitis. Oxid Med Cell Longev. 2016;2016:2721469.

2. Chiu YH, Coull BA, Cohen S, Wooley A, Wright RJ. Prenatal and postnatal maternal stress and wheeze in urban children: effect of maternal sensitization. Am J Respir Crit Care Med 2012;186(2):14754.

3. Wright RJ, Fisher K, Chiu YH, Wright RO, Fein R, Cohen S, Coull BA. Disrupted prenatal maternal cortisol, maternal obesity, and childhood wheezing: insights into prenatal programming. Am J Respir Crit Care Med 2013;187(11):1186-93.

4. Khashan AS, Wicks S, Dalman C, Henriksen TB, Li J, Mortensen PB, Kenny LC. Prenatal stress and risk of asthma hospitalization in the offspring: a Swedish population-based study. Psychosom Med 2012;74(6):635-41.

5. Guxens M, Sonnenschein-van der Voort AM, Tiemeier H, Hofman A, Sunyer J, de Jongste JC, et al. Parental psychological distress during pregnancy and wheezing in preschool children: the Generation R Study. J Allergy Clin Immunol 2014;133(1):59-67.

6. Turcotte-Tremblay AM, Lim R, Laplante DP, Kobzik L, Brunet A, King. Prenatal maternal stress predicts childhood asthma in girls: project ice storm. Biomed Res Int. 2014;2014:201717.

7. de Marco R, Pesce G, Girardi P, Marchetti P, Rava M, Ricci P, Marcon A. Foetal exposure to maternal stressful events increases the risk of having asthma and atopic diseases in childhood. Pediatr Allergy Immunol 2012;23(8):724-29.

8. Peters JL, Cohen S, Staudenmayer J, Hosen J, Platts-Mills TA, Wright RJ. Prenatal negative life events increases cord blood IgE: interactions with dust mite allergen and maternal atopy. Allergy 2012; 67(4):545-51.

9. Medsker B, Forno E, Simhan H, Celedón JC. Prenatal stress, prematurity, and asthma. Obstet Gynecol Surv 2015;70(12):773-79.

10. Herba CM, Glover V, Ramchandani PG, Rondon MB. Maternal depression and mental health in early childhood: an examination of underlying mechanisms in low-income and middle-income countries. Lancet Psychiatry 2016;3(10):983-92.

11. Oberlander TF, Weinberg J, Papsdorf M, Grunau R, Misri S, Devlin AM. Prenatal exposure to maternal depression, neonatal methylation of human glucocorticoid receptor gene (NR3C1) and infant cortisol stress responses. Epigenetics 2008;3(2):97-106.

12. Moisiadis VG, Matthews SG. Glucocorticoids and fetal programming part 1: outcomes. Nat Rev Endocrinol 2014;10(7):391-402.

13. Wang IJ, Wen HJ, Chiang TL, Lin SJ, Chen PC, Guo YL. Maternal employment and atopic dermatitis in children: a prospective cohort study. Br J Dermatol 2013;168(4):794-801.

14. Sidbury R, Davis DM, Cohen DE, Cordoro KM, Berger TG, Bergman JN, et al. Guidelines of care for the management of atopic dermatitis: section 3. Management and treatment with phototherapy and systemic agents. J Am Acad Dermatol 2014;71(2):327-49.

15. Weidinger S, Novak N. Atopic dermatitis. Lancet 2016;387(10023): 1109-22.

16. Andersson N, Hansen M, Larsen A, Hougaard KS, Kolstad HA, Schlünssen V. Prenatal maternal stress and atopic diseases in the 


\section{Maternal Stress and Allergic Diseases in Children}

child: a systematic review of observational human studies. Allergy 2016;71(1):15-26.

17. Garg A, Chren MM, Sands LP, Matsui MS, Marenus KD, Feingold KR, Elias PM. Psychological stress perturbs epidermal permeability barrier homeostasis: implications for the pathogenesis of stress-associated skin disorders. Arch Dermatol 2001;137(1):53-59.

18. Wright RJ, Cohen RT, Cohen S. The impact of stress on the development and expression of atopy. Curr Opin Allergy Clin Immunol 2005;5(1):23-29.

19. Chang HY, Suh DI, Yang SI, Kang MJ, Lee SY, Lee E, et al. Prenatal maternal distress affects atopic dermatitis in offspring mediated by oxidative stress. J Allergy Clin Immunol 2016;138(2):468475.

20. Williams MS, Kwon J. T cell receptor stimulation, reactive oxygen species, and cell signaling. Free Radical Biology and Medicine 2004; 37(8):1144-1151.

21. Wright RJ, Schreier HM. Seeking an integrated approach to assessing stress mechanisms related to asthma: is the allostatic load framework useful? Am J Respir Crit Care Med 2013;187(2):115-116.

22. Wright RJ. Prenatal maternal stress and early caregiving experiences: implications for childhood asthma risk. Paediatr Perinat Epidemiol 2007;21(Suppl 3):8-14.

23. Polańska K, Hanke W, Gromadzińska J, Ligocka D, Gulczyńska E, Sobala W, Wásowicz W. Polish mother and child cohort study: defining the problem, the aim of the study and methodological assumption. Int J Occup Med Environ Health 2009;22(4):383-91.

24. Polanska K, Hanke W, Jurewicz J, Sobala W, Madsen C, Nafstad P, Magnus P. Polish mother and child cohort study (REPRO_PL): methodology of follow-up of the children. Int J Occup Med Environ Health 2011;24(4):391-98.

25. Polanska K, Krol A, Merecz-Kot D, Jurewicz J, Makowiec-Dabrowska T, Chiarotti F, et al. Maternal stress during pregnancy and neurodevelopmental outcomes of children during the first 2 years of life. J Paediatr Child Health 2017;53(3):263-70.

26. Polańska K, Hanke W, Król A, Sobala W, Madsen C, Nafstad P, Magnus P. Polish mother and child cohort study (REPRO_PL): methodology of the follow-up of the children at the age of 7. Int J Occup Med Environ Health 2016;29:883-93.

27. Stelmach I, Majak P, Jerzynska J, Podlecka D, Stelmach W, Polańska $\mathrm{K}$, et al. Cord serum 25-hydroxyvitamin D correlates with early childhood viral-induced wheezing. Respir Med 2015;109(1):38-43.

28. Stelmach I, Majak P, Jerzynska J, Podlecka D, Stelmach W, Polańska $\mathrm{K}$, et al. The effect of prenatal exposure to phthalates on food allergy and early eczema in inner-city children. Allergy Asthma Proc 2015; 36(4):72-78.

29. Stelmach I, Grzelewski T, Bobrowska-Korzeniowska M, Kopka M, Majak P, Jerzynska J, et al. The role of zinc, copper, plasma glutathione peroxidase enzyme, and vitamins in the development of al- lergic diseases in early childhood: The Polish mother and child cohort study. Allergy Asthma Proc 2014;35(3):227-232.

30. Stelmach I, Bobrowska-Korzeniowska M, Smejda K, Majak P, Jerzynska J, Stelmach W, et al. Risk factors for the development of atopic dermatitis and early wheeze. Allergy Asthma Proc 2014;35(5): 382-89.

31. Dudek B, Waszkowska M, Merecz D, Hanke W. Employees' protection against occupational stress. 2nd ed. Nofer Institute of Occupational Medicine, Lodz, Poland. 2004.

32. Cohen S, Kamarck T, Mermelstein R. A global measure of perceived stress. J Health Soc Behav 1983;24(4):385-96.

33. Holmes TH, Rahe RH. Social readjustment rating scale. J Psychosom Res 1967;11(2):213-18.

34. Trump S, Bieg M, Gu Z, Thürmann L, Thürmann L, Bauer T, et al. Prenatal maternal stress and wheeze in children: novel insights into epigenetic regulation. Sci Rep 2016;28:6:28616.

35. Hartwig IR, Sly PD, Schmidt LA, van Lieshout RJ, Bienenstock J, Holt PG, Arck PC. Prenatal adverse life events increase the risk for atopic diseases in children, which is enhanced in the absence of a maternal atopic predisposition. J Allergy Clin Immunol 2014;134(1): 160-69.

36. Liu X, Olsen J, Agerbo E, Yuan W, Sigsgaard T, Li J. Prenatal stress and childhood asthma in the offspring: role of age at onset. Eur J Public Health 2015;25(6):1042-46.

37. Rosa MJ, Just AC, Tamayo Y Ortiz M, Schnaas L, Svensson K, Wright RO, et al. Prenatal and postnatal stress and wheeze in Mexican children: sex-specific differences. Ann Allergy Asthma Immunol 2016;116(4):306-12.

38. Polloni L, Ferruzza E, Ronconi L, Lazzarotto F, Toniolo A, Bonaguro R, Muraro A. Perinatal stress and food allergy: a preliminary study on maternal reports. Psychol Health Med 2015;20(6):732-41.

39. Larsen AD, Schlunssen V, Christensen BH, Bonde JP, Obel C, Thulstrup AM, et al. Exposure to psychosocial job strain during pregnancy and odds of asthma and atopic dermatitis among 7-year old children: a prospective cohort study. Scand J Work Environ Health 2014;40(6):639-48

40. Wen HJ, Wang YJ, Lin YC, Chang CC, Shieh CC, Lung FW, Guo YL. Prediction of atopic dermatitis in 2-yr-old children by cord blood IgE, genetic polymorphisms in cytokine genes, and maternal mentality during pregnancy. Pediatr Allergy Immunol 2011;22(7): 695-703.

41. Karlsson L, Nousiainen N, Scheinin NM, Maksimow M, Salmi M, Lehto SM, et al. Cytokine profile and maternal depression and anxiety symptoms in mid-pregnancy: the FinnBrain Birth Cohort Study. Arch Womens Ment Health 2017;20(1):39-48.

42. Tsuji M, Koriyama C, Yamamoto M, Anan A, Shibata E, Kawamoto T. The association between maternal psychological stress and inflammatory cytokines in allergic young children. PeerJ. 2016;18:4: e1585. 